\title{
Atypical phenotype of an old disease or typical phenotype of a new disease: deficiency of adenosine deaminase 2
}

\author{
Mustafa Çakan ${ }^{1}$, Nuray Aktay-Ayaz ${ }^{1}$, Şerife Gül Karadağ ${ }^{1}$, Eda Tahir-Turanlı², \\ Kelsey Stafstrom ${ }^{3}$, Wayne Bainter ${ }^{3}$, Raif S. Geha ${ }^{3}$, Janet Chou ${ }^{3}$ \\ ${ }^{1}$ Clinic of Pediatric Rheumatology, Kanuni Sultan Süleyman Research and Training Hospital, ${ }^{2}$ Department of Molecular \\ Biology and Genetics, Istanbul Technical University, Istanbul, Turkey, ${ }^{3}$ Division of Immunology, Boston Children's Hospital \\ and Department of Pediatrics, Harvard Medical School, Boston, USA. E-mail: mustafacakan@hotmail.com \\ Received: 26th January 2018, Accepted: 1st March 2018
}

SUMMARY: Çakan M, Aktay-Ayaz N, Karadağ ŞG, Tahir-Turanlı E, Stafstrom K, Bainter W, Geha RS, Chou J. Atypical phenotype of an old disease or typical phenotype of a new disease: deficiency of adenosine deaminase 2. Turk J Pediatr 2019; 61: 413-417.

Deficiency of adenosine deaminase 2 (DADA2) is an autosomal recessive autoinflammatory disorder caused by mutations in CECR 1 (cat eye syndrome chromosome region, canditate 1) gene, which encodes the enzyme adenosine deaminase 2 necessary for endothelial cell survival and function.

The diversity of the clinical phenotypes associated with DADA2 include polyarteritis nodosa-like vasculitic features, early-onset stroke, mild to severe immunodeficiency and cytopenias.

The diagnosis of the disease may be difficult due to complex clinical phenotype. Herein, we present a case of DADA2 presenting with vasculitis, amarousis fugax, gastrointestinal bleeding and silent lacunar infarct successfully treated with etanercept.

Key words: amaurosis fugax, deficiency of adenosine deaminase 2, stroke, vasculitis.

Deficiency of adenosine deaminase 2 (DADA2) is a recently described autoinflammatory disorder characterized by signs and symptoms of systemic vasculitis and inflammation including early onset stroke, livedoid skin rash, and recurrent fevers. ${ }^{1-3}$ The disease is caused by autosomal recessive loss-of-function mutations in the CECR1 (cat eye syndrome chromosome region, canditate 1) gene, which encodes adenosine deaminase 2 (ADA2) enzyme $^{1,2}$. ADA2 induces proliferation of monocytes and enhances differentiation of macrophages toward the anti-inflammatory M2 subset instead of proinflammatory M1 subset. It is also thought that ADA2 acts as a growth factor for endothelial cells and is responsible for the integrity of vascular endothelial cells. ${ }^{1-4}$

Many patients with DADA2 present with vasculitis, notably a polyarteritis nodosa (PAN)- like phenotype including livedo reticularis/ racemosa, digital gangrene, hypertension, ischemic/hemorrhagic stroke, gastrointestinal manifestations and neuropathies. ${ }^{1,2}$ There are reports of patients with DADA2 who have features of immunodeficiency or hematological abnormalities (i.e., neutropenia, anemia), either in the presence or absence of vasculitis. $^{5-8}$ This phenotypic variability can delay the diagnosis of DADA2.

\section{Case Report}

A 15-year-old boy was admitted to the rheumatology clinic with the complaints of fever, vomiting, diarrhea, diffuse arthralgia and myalgia for 5 days. He was the fifth child of healthy, consanguineous parents and had a healthy dizygotic twin brother. 
The patient was well until 12 years of age, when he presented with painful, indurated, pinkish rash on the legs that lasted for less than a year and healed with minimal hyperpigmentation. He was also describing Raynaud phenomenon for the last three years. The clinical picture was complicated with myalgia and arthralgia attacks for the last 2 years, followed by diarrhea, abdominal pain, headache, and fever attacks lasting for 3-5 days and recurring every 2-3 weeks in the last 6 months. And for the last 3 months, he was describing loss of vision in one eye resolving in less than one minute every other week. Vision loss attacks were independent of fever attacks and some were accompanied by headaches but both eyes were never involved at the same time. He was denying any genital ulceration but had oral aphthosis two or three times per year.

Physical examination was remarkable for livedo reticularis rash and Raynaud phenomenon without accompanying arthritis, hepatosplenomegaly, or lymphadenopathy. $\mathrm{He}$ did not have any sensory or motor deficit and mental development was normal. Laboratory evaluation revealed anemia (hemoglobin: $8.9 \mathrm{~g}$ / $\mathrm{dl}$ ), elevated acute phase reactants [C-reactive protein: $111 \mathrm{mg} / \mathrm{L}$ (normal: 0-5 $\mathrm{mg} / \mathrm{L}$ ), erythrocyte sedimentation rate: $88 \mathrm{~mm} / \mathrm{hr}$ (normal: 0-15 mm/hr), serum amyloid A: 528 $\mathrm{mg} / \mathrm{L}$ (normal: 0-6.4 mg/L)] with normal liver and kidney function tests. Extractable nuclear antigen (ENA) and antineutrophil cytoplasmic antibody (ANCA) profile were negative and serum immunoglobulin levels were normal except for low IgM (serum IgM: $44.6 \mathrm{mg}$ / $\mathrm{dl}$, normal: $83-282 \mathrm{mg} / \mathrm{dl}$ ). Eye examination and ocular angiography were normal without any explanatory findings for amaurosis fugax attacks. Cranial magnetic resonance (MR) angiography was normal but cranial MR imaging showed a lacunar infarct in the left pons region (Fig. 1). Later in the course, he developed severe abdominal pain, diarrhea and massive hematochezia requiring red blood cell transfusions. Upper and lower gastrointestinal endoscopy revealed normal appearing mucosa and thoraco-abdominal MR angiography was normal too. Pathological examination of gastrointestinal biopsy specimens showed chronic mucosal inflammation with eosinophils throughout the colon.

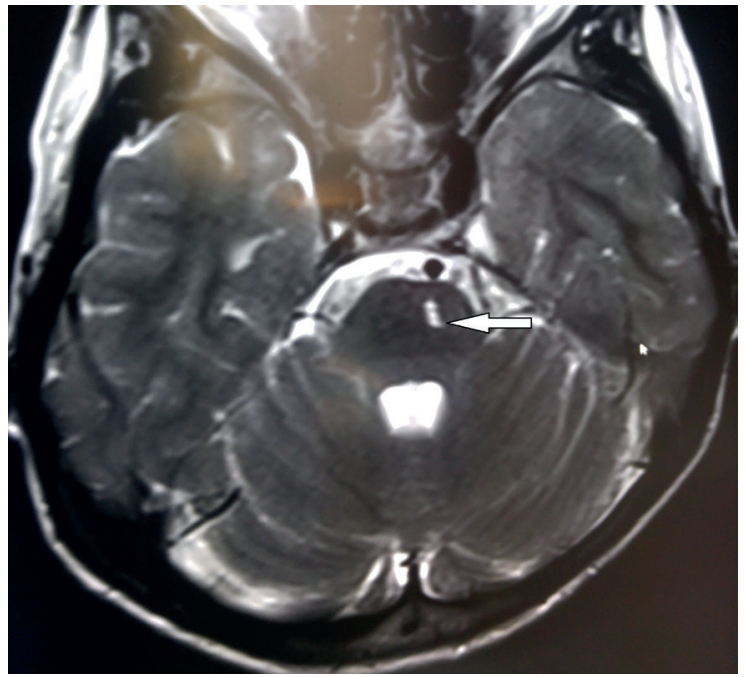

Fig. 1. Lacunar infarct in the left brain stem (white arrow)

Genetic testing for Mediterranean fever (MEFV) was negative. Pathergy testing and HLA-B51 analysis were positive, prompting treatment for possible Behçet's disease with colchicine, prednisolone and mesalazine. Under 1.5 months of this treatment fever, diarrhea and amaurosis fugax attacks did not show any improvement and infliximab was added to the regimen. During the second infusion of infliximab, he developed hypertension for the first time that persisted afterwards so nifedipine and enalapril were added.

Re-evaluation of his clinical features, namely the recurrent fevers, livedo reticularis, Raynaud phenomenon, hypertension, lacunar infarct in the brain stem, abdominal pain attacks, hematochezia, ocular findings, high acute phase reactants, and low IgM led to the suspicion of DADA2. Simultaneously, next-generation sequencing for a panel of 264 genes associated with host immunity identified a homozygous mutation in CECR 1 (NM_001282225 c.139G >A; p.Gly47Arg), which was confirmed by Sanger sequencing. This mutation has been previously reported to be pathogenic, resulting in early onset stroke and vasculopathy. ${ }^{1,2}$ His healthy father, mother, and brothers were all heterozygous for the same mutation (Fig. 2). Incidentally, a second, heterozygous synonymous mutation in CECR1 (NM_011282225 c.138G>C; p. Lys46Lys) was found in only the proband's 


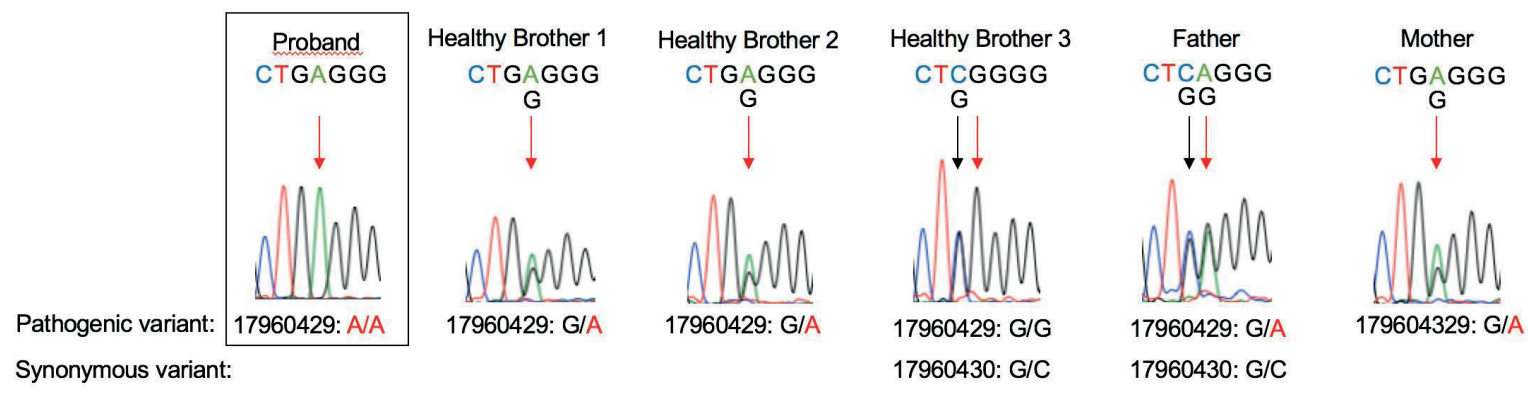

Fig. 2. Sanger sequencing of the pathogenic CECR1 (NM_011282225 c.139G>A; p.Gly47Arg) mutation in the proband and family members (noted with a red arrow). The black arrow notes a synonymous heterozygous mutation in CECR 1 incidentally found in only the healthy father and healthy brother 3 , which does not segregate with the pathogenic variant and is absent in the proband.

father and healthy brother (Fig. 2). This incidental mutation did not segregate with the pathogenic p.Gly47Arg variant, preserved the wild-type amino acid sequence, and was found only in healthy carriers, thus demonstrating that it does not contribute to the proband's disease. With the diagnosis of DADA2, he was maintained on etanercept (25 mg/sc/week), fresh frozen plasma (FFP) infusions $(15 \mathrm{ml} /$ $\mathrm{kg}$, every two weeks) and anti-hypertensives, resulting in dramatic clinical improvement. Anti-hypertensives and FFP infusions were discontinued after 6 months. He has not had fever, diarrhea or ocular attack since the first dose of etanercept 3 years ago.

Informed consent was received from the family.

\section{Discussion}

Mechanisms driving autoinflammatory diseases have been elucidated by advances in sequencing technologies and international collaborations and many new disorders have been described recently. $4,9,10$ DADA2 was described in 2014 and initial cohorts consisted of children with early onset stroke and PANlike vasculitic features and most of the cases had diagnosis of PAN or undefined vasculitis/ autoinflammatory syndrome. ${ }^{1,2}$ Subsequent reports have shown that this disease can be associated with anemia, neutropenia, mild to severe immune deficiency, thus expanding the clinical spectrum of DADA2. ${ }^{1,2,6,11-15}$

The clinical spectrum of the disease ranges from mild skin involvement to severe major organ involvement that may lead to mortality. Most of the patients have constitutional symptoms like recurrent fevers, myalgia and arthralgia as a common feature. ${ }^{1,2,12,14}$ Recurrent fevers may lead to consideration of a periodic fever syndrome, especially of familial Mediterranean fever (FMF), in countries where FMF is a prevalent disease. ${ }^{11,14,16}$

Skin manifestations of DADA2 include livedo reticularis, livedo racemosa, maculopapular rashes, erythema nodosum, Raynaud phenomenon, ulcers, and digital gangrene. ${ }^{1-3}$ Nearly all of the cases described in the literature have one of these skin manifestations, with livedo reticularis being the most common..$^{1-3,12,14}$ A few cases had only skin involvement and were diagnosed as cutaneous PAN, reflecting the degree of clinical diversity of DADA2. ${ }^{13}$ Our case had Raynaud phenomenon, livedo reticularis and erythema nodosum, but no other clinical or laboratory evidence of a connective tissue disease.

Ocular involvement may occur as external ocular muscle palsies leading to diplopia and strabismus, optic nerve atrophy, uveitis, and occlusive retinal vasculitis leading to monocular or binocular permanent vision loss. ${ }^{1,6,13,14}$ Our case had unilateral amaurosis fugax attacks that were possibly secondary to vasospasm of the retinal artery, since no vascular abnormalities by ocular angiography were identified. To our knowledge, this is the first description of amaurosis fugax attacks in DADA2.

Central nervous system (CNS) and gastrointestinal system (GIS) involvement are the leading causes of morbidity and mortality in DADA2., ${ }^{1,2,6,14}$ CNS involvement 
may be reflected as transient ischemic attacks, ischemic or hemorrhagic lacunar strokes, and intraventricular hemorrhage. Some patients may have clinically silent episodes, as seen in our case, and some of them may have permanent disability or death. ${ }^{1,2,11-14,17}$ The symptoms and signs of GIS involvement are secondary to mesenteric vasculitis and ischemia, resulting in recurrent abdominal pain, diarrhea, hepatosplenomegaly, pancreatitis, portal hypertension, hematochezia, or bowel perforation. ${ }^{1,2,12-14}$ Our case had recurrent abdominal pain and diarrhea attacks complicated by massive bleeding.

Most of the DADA2 cases had initial diagnosis of PAN. ${ }^{1,2,14,15}$ We did not suspect PAN in this case, because he did not have hypertension at the early stages of the disease, and neither had renal involvement nor neuropathy. Also we were unable to demonstrate any vascular abnormality in MR angiographies. Stenosis and/or aneurysms of middle sized arteries like renal, mesenteric, hepatic and celiac arteries are usual findings both in PAN and DADA2., ${ }^{1,2,6}$ Our working diagnosis was Behçet's disease because of the recurrent fevers, oral aphthosis, GIS and CNS manifestations, amaurosis fugax attacks, pathergy and HLA-B51 positivity. ${ }^{18}$ Unresponsiveness of the patient to the treatment made us reconsider the diagnosis.

Treatment with tumor necrosis factor (TNF) $-\alpha$ antagonists (i.e., etanercept, adalimumab, infliximab) have been shown to be effective for DADA2. ${ }^{1,2,12-15}$ Notably, the patient responded well to etanercept, but not infliximab, indicating the utility for empirical use of different TNF- $\alpha$ antagonists in this disease.

In conclusion, DADA2 should be considered in the differential diagnosis of patients with signs and symptoms of unexplained periodic fever syndrome, vasculitis, stroke, immunodeficiency, or cytopenia. If a patient has forme fruste form of an old, well-known disease, or does not respond to conventional treatment, they probably have another or possibly newly described disease.

\section{Acknowledgement}

We thank the technical staff of both Istanbul Technical University (Ayşe Balaban) and Boston Children Hospital for their assistance in sequencing.

\section{REFERENCES}

1. Zhou Q, Yang D, Ombrello AK, et al. Early-onset stroke and vasculopathy associated with mutations in ADA2. N Engl J Med 2014; 370: 911-920.

2. Navon Elkan P, Pierce SB, Segel R, et al. Mutant adenosine deaminase 2 in a polyarteritis nodosa vasculopathy. N Engl J Med 2014; 370: 921-931.

3. Caorsi R, Penco F, Schena F, Gattorno M. Monogenic polyarteritis: The lesson of ADA2 deficiency. Pediatr Rheumatol Online J 2016; 14:51.

4. Stoffels M, Kastner DL. Old dogs, new tricks: Monogenic autoinflammatory disease unleashed. Annu Rev Genomics Hum Genet 2016; 17: 245-272.

5. Van Eyck Jr L, Hershfield MS, Pombal D, et al Hematopoietic stem cell transplantation rescues the immunologic phenotype and prevents vasculopathy in patients with adenosine deaminase 2 deficiency. J Allergy Clin Immunol 2015; 135: 283-287.e5.

6. Van Montfrans JM, Hartman EA, Braun KP, et al. Phenotypic variability in patients with ADA2 deficiency due to identical homozygous R169Q mutations. Rheumatology (Oxford) 2016; 55: 902910.

7. Hsu AP, West RR, Calvo KR, et al. Adenosine deaminase type 2 deficiency masquerading as GATA2 deficiency: Successful hematopoietic stem cell transplantation. J Allergy Clin Immunol 2016; 138: 628-630.e2.

8. Hashem H, Egler R, Dalal J. Refractory pure red cell aplasia manifesting as deficiency of adenosine deaminase 2. J Pediatr Hematol Oncol 2017; 39: e293-e296.

9. de Jesus AA, Goldbach-Mansky R. Newly recognized Mendelian disorders with rheumatic manifestations. Curr Opin Rheumatol 2015; 27: 511-519.

10. Aksentijevich I. Update on genetics and pathogenesis of autoinflammatory diseases: The last 2 years. Semin Immunopathol 2015; 37: 395-401.

11. Garg N, Kasapcopur O, Foster J 2nd, et al. Novel adenosine deaminase 2 mutations in a child with a fatal vasculopathy. Eur J Pediatr 2014; 173: 827-830.

12. Batu ED, Karadag O, Taskiran EZ, et al. A case series of adenosine deaminase 2-deficient patients emphasizing treatment and genotype-phenotype correlations. J Rheumatol 2015; 42: 1532-1534. 
13. Nanthapisal S, Murphy C, Omoyinmi E, et al. Deficiency of adenosine deaminase type 2: A description of phenotype and genotype in fifteen cases. Arthritis Rheumatol 2016; 68: 2314-2322.

14. Sahin S, Adrovic A, Barut K, et al. Clinical, imaging and genotypical features of three deceased and five surviving cases with ADA2 deficiency. Rheumatol Int 2018; 38: 129-136

15. Caorsi R, Penco F, Grossi A, et al. ADA2 deficiency (DADA2) as an unrecognised cause of early onset polyarteritis nodosa and stroke: A multicentre national study. Ann Rheum Dis 2017; 76: 1648-1656.
16. Nihira $\mathrm{H}$, Nakagawa K, Izawa $\mathrm{K}$, et al. Fever of unknown origin with rashes in early infancy is indicative of adenosine deaminase type 2 deficiency. Scand J Rheumatol 2018; 47: 170-172.

17. Poswar Fde O, da Fonseca RM, de Albuquerque LC, et al. Adenosine deaminase 2 deficiency presenting as spastic paraplegia and systemic vasculitis. J Neurol 2016; 263: 818-820.

18. Pektaş A, Devrim I, Beşbaş N, Bilginer Y, Cengiz AB Ozen S. A child with Behçet's disease presenting with a spectrum of inflammatory manifestations including epididymoorchitis. Turk J Pediatr 2008; 50: 78-80. 